\title{
Evaluation of Handover Activities from the Perspective of the System Acquisition and Front-End Support
}

\author{
Kaleem Ullah, Ahmad Salman Khan, Mira Kajko-Mattsson \\ School of Information and Communication Technology \\ The Royal Institute of Technology (KTH) \\ Stockholm, Sweden \\ kaleemu@kth.se, askhan@kth.se,mekm2@kth.se
}

\begin{abstract}
Even if software handover is a critical process in the lifecycle of a software system, there is still very little research done within its domain. In this paper, we evaluate the taxonomy of handover activities as defined in $\mathrm{EM}^{3}$ : Handover Process Model. We do it in an industrial setting in a context where a software system is handed over from a vendor conducting system development, evolution and maintenance to an acquirer using the system and conducting front-end support. Our results show that the taxonomy is realistic in the context studied. However, it misses some important deployment and training activities.
\end{abstract}

Keywords- transition; deployment; acquisition; version and configuration management; documentation; maintainability

\section{INTRODUCTION}

Transferring control from one party to another is a difficult task. The difficulty varies depending on what is transferred, by who it is transferred and when it is transferred [2][5]. Even the handover of a simple product, such as for instance, handover of a telephone from the vendor to the end-user may imply that one does not only transfer the product, but also all the knowledge about the product, the knowledge about the technology used, the responsibility for operating the product, and the responsibility for managing the product throughout its product lifecycle.

Handover of a software system is a very complex and multifaceted activity. Here, the complexity strongly varies depending on the system complexity and criticality, number of parties involved, technology used for implementing and supporting the system, documentation requirements, quality requirements, security and safety requirements and many other important factors.

Irrespective of whether the transferred systems is complex or not and irrespective of the number of parties involved in transitioning it, a software system handover is always a critical activity in the software lifecycle process. If not properly prepared and managed, it may jeopardize the system delivery and undermine the credibility of the organizations involved in it. Despite this, there are very few handover process models today. These are [7][8][9]. Currently, a handover process model being developed is $E M^{3}$ : Handover Process Model. EM stand for Evolution and Maintenance Management Model [6]. Right now, it only contains a taxonomy of handover activities [1][2][3].

Software system handover or transition is mainly defined as a controlled and coordinated activity during which a software system is handed over from the team performing software development to the team performing post-development maintenance and support [8]. Some studies, however, have revealed that software handover may also take place in other contexts, that is, between other phases and parties [2][5]. One such context may be the handover of system responsibilities from the vendor to the system acquirer.

In this paper, we evaluate the taxonomy activities as defined in $E M^{3}$ : Handover Process Model in an industrial setting. The context studied covers the handover of a software system from the vendor conducting system development, evolution and maintenance to an acquirer organization using the end product and conducting front-end support [4]. The study is made from the acquirer's and front-end support perspective within VAST Pakistan. Due to sensitivity of the results presented herein, we do not display the company's real name. Instead, we use its fictive name, which is VAST Pakistan.

The remainder of the paper is as follows. Section II describes method steps taken to conduct the study and the validity of our results. Section III briefly presents the handover taxonomy. Section IV presents the results of the taxonomy evaluation. Finally, Sections V and VI make final remarks.

\section{METHOD}

In this section, we present our method. In Section II.A, we describe the steps taken when conducting this study. In Section II.B, we describe the validity of our results.

\section{A. Research Steps}

We conducted our study in three major steps. These are: (1) Confirmation of the Handover Process, (2) Taxonomy Evaluation, and (3) Taxonomy Improvement. 
We have been offered the opportunity to study a handover process at VAST Pakistan. Due to the fact, however, that the handover process has many strongly varying contexts [2][5], we evaluated first whether the handover process at VAST Pakistan was relevant for our study. For this reason, as a first step, we conducted a series of informal and open-ended interviews whose purpose was to validate the context of the process. This step ended in (1) a confirmation that the context was right for our study, (2) a general understanding of the handover process at VAST Pakistan, and (3) a discovery of new handover activities.

In the Taxonomy Evaluation phase, we studied the handover process at VAST Pakistan. Here, we compared the activities in $E M^{3}$ : Handover Process $M o d e l$ with its industrial correspondences at VAST Pakistan. We did it by conducting a series of open-ended interviews and discussions using very simple questionnaires. For each activity, we simply asked (1) whether the activity was performed (2) what role performed it and (3) where in the lifecycle phase it was performed.

For each component, we verified the need and usefulness of its activities in the organization's transition process. An activity was valid if it was performed. If it was not performed, we investigated the reasons behind. Also, we looked for the activities that were performed at the organization, but not presented in the $E M^{3}$ taxonomy. Finally, in the Taxonomy Improvement step, we added new activities to our taxonomy and modified the existing ones.

\section{B. Validity}

We conducted our study in a real-world industrial setting. VAST Pakistan is one of the largest mobile operators in Pakistan. It is part of VAST, which is a mobile communication service provider in 14 countries across Europe and Asia.

VAST Pakistan has been involved in a handover process for many years. Its role is mainly that of an acquirer. It acquires large software systems from a third party who develops, evolves and maintains them. VAST Pakistan only uses the system and provides $1^{\text {st }}$ and $2^{\text {nd }}$ line support on these systems [4].

Our contact person and interviewee at VAST Pakistan was a Business Support System (BSS) executive. The interviewee has been involved in multiple system acquisition processes for three years. He is part of the team that is responsible for executing the handover and for supporting the system during the post-delivery phase.

All case studies encounter validity threats. The main external validity threat to our study was the fact that our evaluation was made within only one company. Regarding construct validity, the main threat concerned the choice and collection of the right measures for the process being studied. The risk was that the researcher misinterpreted the handover process. In order to minimize this threat, the process of understanding VAST Pakistan's handover process was conducted in an iterative manner during which the interviewer continuously evaluated the elicited information with the interviewee. Finally, the internal validity is not relevant for our context due to the exploratory nature of our study.

\section{TAXONOMY OF HANDOVER ACTIVITIES}

$E M^{3}$ : Handover Process Model categorizes the handover activities into seven different components [1]. As shown in Figure 2, these are: Management and Administration, Maintenance Environment, Version and Configuration Management, Deployment, Training, Documentation, and Maintainability. Their activities are all listed in Tables I-VII.

The Management and Administration component describes the activities required for managing and administering the handover process. As shown in Table I, the activities in this component concern (1) identification of maintenance organization, (2) establishment of a transition team, (3) creation of a transition plan, and (4) development of management plans.

The Maintenance Environment component includes activities required for setting up the maintenance environment, installing the system and its data, and handling the modification requests. The maintenance environment for a software system comprises hardware and software suites and maintenance support suites. As listed in Table II, the component covers activities dealing with the (1) determination/assessment of the need and installation/upgrade of hardware/software suite, (2) determination and assessment of maintenance support suite and supplement of support suite, (3) installation of software support suite, (4) installation of software baseline and data, (5) transfer of modification requests from the developer to the maintainer, and (6) placement of modification requests in a Modification Request Repository (MRR).

The Version and Configuration Management component consists of the activities needed for tracking and controlling changes in the transitioned system. As shown in Table III, these activities concern the (1) establishment of software configuration baseline, (2) placement of software under Software Configuration Management (SCM), and (3) placement of software under version control.

The Deployment component consists of activities required for the developer or maintainer to deploy the software system at the acquirer's site. As shown in Table IV, the component covers activities dealing with (1) development of installation procedures, (2) installation of the system, and (3) planning of the future releases.

The Training component addresses the issue of transfer of knowledge. The system stakeholders cannot perform their duties without knowing the system's structural and operational details. Since the maintainer is the main stakeholder for the system, the Training component focuses on the maintainer. As shown in Figure V, it covers training (1) on system, its structure, and operation, (2) on the maintenance processes, (3) on the system support processes, (4) on new technology, and (5) provision of on-site support.

The Documentation component describes the activities for handing over the system documentation and handling it after the system handover. As shown in Table VI, it deals with (1) establishment of a System Documentation Repository (SDR), (2) definition of services to be provided by the SDR, (3) updation of SDR as per SCM, and (4) establishment of standards for documentation development. 


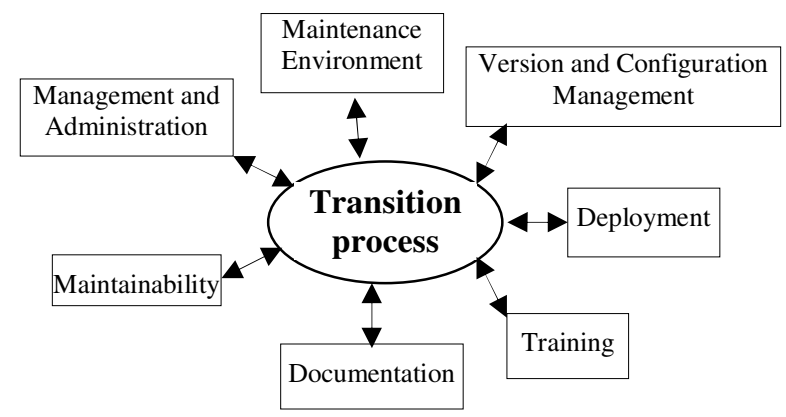

Figure 1. Components of $\mathrm{EM}^{3}$ handover taxonomy [1]

The Maintainability component covers activities for managing system maintainability. As shown in Table VII, the component includes activities dealing with the (1) assessment of system maintainability, and (2) assessment of data maintainability.

\section{TAXONOMY EVALAUTION RESUlTS}

In this section, we present our evaluation results. All components and their status states within the industry are presented in Tables I-VII.

\section{A. Management and Administration}

VAST Pakistan performs all the activities as specified in the Management and Administration component, see Table I. The maintenance organization is identified at the start of system acquisition process (see Activity MA1 in Table I). It is the VAST Pakistan's vendor company. This company develops, evolves and maintains the system for VAST Pakistan throughout its lifecycle. For this reason, the identification of the maintenance team is made by default. This team continuously accepts system requirements as defined by the VAST Pakistan's business team and architects.

A transition team is established very early (see Activity MA2 in Table I). However, its constellation varies with time. Initially, it consists of vendor's high level management personnel and VAST Pakistan's architects and operations team. Architects plan for the system acquisition while the operations team performs the system handover. At VAST Pakistan, the operations team is responsible for supporting a specific system. It corresponds to $1^{\text {st }}$ line and $2^{\text {nd }}$ line support personnel.

With time, the transition team gets expanded with more teams. These include but are not limited to (1) system team, (2) database team, (3) Value Added Services (VAS) team, and (4) Customer Relationship Management (CRM) team. These teams facilitate the transition process by providing support for their related interfaces and by co-coordinating with the maintenance team. The expansion mainly takes place after User Acceptance Testing (UAT).

VAST Pakistan creates a detailed transition plan during the requirements specification phase (see Activity MA3 in Table I). This plan is mainly realized by the operations team. It is the operations team who is responsible for making sure that the transition plan is followed by all the roles and teams involved in the process within the timeframe as planned beforehand.
TABLE I. MANAGEMENT AND ADMINISTRATION. $\checkmark$ STANDS FOR PERFORMED.

\begin{tabular}{|l|l|c|}
\hline MA1 & Identify maintenance organization. & $\checkmark$ \\
\hline MA2 & Establish a transition team. & $\checkmark$ \\
\hline MA3 & Create a transition plan. & $\checkmark$ \\
\hline MA3.1 & Determine transition budget. & $\checkmark$ \\
\hline MA3.2 & Create a transition schedule. & $\checkmark$ \\
\hline MA3.3 & Establish transition procedures. & $\checkmark$ \\
\hline MA3.4 & Define transition resource requirements. & $\checkmark$ \\
\hline MA3.4.1 & Define maintenance manpower requirements. & $\checkmark$ \\
\hline MA3.4.2 & Define maintenance facility requirements. & $\checkmark$ \\
\hline MA4 & Develop management plans. & $\checkmark$ \\
\hline
\end{tabular}

Regarding the transition budget (see Activity MA3.1 in Table I), it is part of the overall system budget. It is determined by the architects and business team who estimate the system costs such as software, hardware, training and the handover cost. Regarding the transition schedule (see Activity MA3.2 in Table I), its timeframe is planned and strictly followed. Standard operating rules and procedures for managing and coordinating the transition process are defined beforehand (see Activity MA3.3 in Table I).

Manpower resources and facility requirements are well planned in advance (see Activities MA3.4.1-3.4.2 in Table I). The manpower resource planning is supported by documents recording team member name, role, department, responsibilities, activities assigned to perform the handover related tasks, and timeframe allowed for a certain activity. Facility inventory requirements document include assessment of facilities required and facility acquisition details.

VAST Pakistan develops various management plans (see Activity MA4 in Table I). These plans are SCM, testing, and training plans. SCM and software testing plans are made when the system development is complete. Developer/vendor establishes the baseline for SCM and VAST Pakistan's maintenance team plans for the advanced SCM functionality. Regarding software testing plans, they carefully identify and include all the teams having touch-points with the transitioned system. Finally, training plans identify training needs and ways of providing training.

\section{B. Maintenance Environment}

VAST Pakistan conducts some of the activities as defined in the Maintenance Environment component, see Table II. It determines/assesses the hardware, software and support suites and installs the software and hardware suites. Vendor installs the support suite and support software. However, VAST Pakistan does not manage modification requests or perform installation of software and data for maintenance purposes.

Determination of hardware and software suites (see Activities ME1-ME4 in Table II) is already conducted during the system requirements specification phase. The VAST Pakistan architects specify the details of current hardware, software, database and related system components and convey them to the vendor. The vendor then assesses the needs for the new system and recommends the optimal hardware and software solutions. The recommended suite is then installed by VAST Pakistan. 
TABLE II. MAINTENANCE ENVIRONMENT. $\checkmark$ STANDS FOR PERFORMED. $\times$ STANDS FOR NOT PERFOMED. A STANDS FOR ASSISTS THE VENDOR

\begin{tabular}{|c|c|c|}
\hline ME1 & Determine hardware/software suite needs & $\checkmark$ \\
\hline ME2 & Install hardware/software suite & $\checkmark$ \\
\hline ME3 & Assess current hardware/software suite, if any & $\checkmark$ \\
\hline ME4 & Remedy the deficiencies in the HW/SW suite, if any & $\checkmark$ \\
\hline ME5 & Determine/assess maintenance support suite & $\checkmark$ \\
\hline ME6 & Supplement maintenance support suite with new tools & A \\
\hline ME7 & Install support software & A \\
\hline ME8 & Install software baseline & $x$ \\
\hline ME9 & Install data & $x$ \\
\hline ME10 & $\begin{array}{l}\text { Transfer modification requests from development to } \\
\text { maintenance }\end{array}$ & $x$ \\
\hline ME11 & Place modification requests in the MRR & $x$ \\
\hline
\end{tabular}

Determining, assessing and installing the maintenance support suite is slightly different (see Activities ME5-ME6 in Table II). The vendor recommends acquisition of appropriate tools provides them and sets them up. Same is the case with installation of the support software (see Activity ME7 in Table II). The vendor provides and installs the support software.

VAST Pakistan does not need to perform the system and data installation activities due to its acquirer role (see Activities ME8-ME9 in Table II). The system and data installation at VAST's site is performed as part of system deployment, see Section IV.D.

Being the system acquirer, VAST Pakistan does not transfer any modification requests from development to maintenance. Neither does it place modification requests in the MRR (see Activities ME10-ME11 in Table II). However, VAST Pakistan creates Change Requests (CRs) during the transition. These change requests report on problems or suggest minor changes to be resolved or made during the transition phase. Although the CR documents are prepared with great care and are very detailed, their management is mainly manual. CR owners keep track of their CR documents and their management during the transition phase. The CRs are not centrally stored and available for the whole organization.

\section{Version and Configuration Management}

VAST Pakistan performs only a single activity in the Version and Configuration Management component, see Table III. It is the vendor who creates a configuration baseline after the system is deployed while VAST Pakistan puts it under SCM (see Activities VC1-VC2 in Table III). VAST Pakistan only takes over the system, optimizes the configuration, adapts it to its own needs, and makes the system operational.

During the system deployment, two different versions of the system exist: (1) the original system on the vendor's site, and (2) the operational system on the front-end support site at VAST Pakistan. The operational system is configured to meet the front-end support needs on $1^{\text {st }}$ and $2^{\text {nd }}$ support lines. After the system deployment, when VAST Pakistan signs under system acceptance, the vendor updates its mirror system with all the changes that have taken place during the transition phase. This helps testing, replication of operational issues reported by VAST Pakistan, development of CR according to the VAST Pakistan requirements and the like.
TABLE III. VERSION AND CONFIGURATION MANAGEMENT. A STANDS FOR ASSISTS THE VENDOR. $\checkmark$ STANDS FOR PERFORMED. $\times$ STANDS FOR NOT PERFOMED

\begin{tabular}{|l|l|l|}
\hline VC1 & Establish software configuration baseline & A \\
\hline VC2 & Put software under SCM & $\checkmark$ \\
\hline VC3 & Place software under version control & $\mathbf{x}$ \\
\hline
\end{tabular}

\section{Deployment}

VAST Pakistan performs some of the activities defined in the Deployment component, as shown in Table IV. Rest of the activities are performed by the vendor.

It is the vendor who develops the installation procedures for system deployment (see Activity DP1in Table IV) and the vendor's support team deploys the system at VAST Pakistan's site (see Activity DP2 in Table IV) in the mandatory presence of the VAST Pakistan's operations team. The vendor and the operations team both plan for the future system releases (see Activity DP3 in Table IV). They plan release dates, structure of installation patches and procedures for installation.

Regarding the distribution structure, it is different from the system delivery. As already mentioned in Section IV.C, in the context of newly developed systems, it is the vendor who delivers the system to VAST Pakistan. VAST Pakistan adapts and configures it to its own needs and then the vendor updates his mirror version of the system with all the minor adaptation changes. Regarding minor releases, they are delivered in form of solution patches by the vendor. The VAST Pakistan's operations team then adds the solution patches to the system, performs the detailed testing, and monitor how the system works for a certain period of time. If the solution patch is working fine, it is then made part of the system.

The future releases are generated due to requests for system enhancements, minor system updates or bug fixes. In case of system updates, release notes are managed by the vendor. In case of requests for enhancements or bug fixes, the system teams on the VAST Pakistan site handle the releases notes.

Deployment is a crucial part of the transition process at VAST Pakistan. VAST Pakistan systems need to be up and running in order to provide smooth and reliable services to its millions of customers. For this reason, VAST Pakistan takes active part in creating plans for the system deployment (see Activity DP4 in Table IV). It creates (1) system rollback plan, (2) contingency plan, (3) post-deployment monitoring plan, (4) cutover activity plan, and (5) system outage plan.

TABLE IV. DEPLOYMENT. $\checkmark$ STANDS FOR PERFORMED. A STANDS FOR AsSists THE VENDOR. NEW STANDS FOR MISSING ACTIVITY IN THE $E M^{3}$ TAXONOMY

\begin{tabular}{|l|l|l|}
\hline DP1 & Develop installation procedures & A \\
\hline DP2 & Install system & A \\
\hline DP3 & Plan future releases & $\checkmark$ \\
\hline DP3.1 & Plan updates of future releases & $\checkmark$ \\
\hline DP3.2 & Determine the distribution structure & $\checkmark$ \\
\hline ME3.3 & Determine forms of deploying software & $\checkmark$ \\
\hline DP3.4 & Determine the structure of release notes & $\checkmark$ \\
\hline \multicolumn{2}{|c|}{ NEw DEPLOYMENT AcTIVITIES } \\
\hline DP4 & Plan for the system deployment & New \\
\hline DP5 & Test the deployed system touch-points & New \\
\hline DP6 & Monitor the system operation & New \\
\hline DP7 & Install operational data & New \\
\hline
\end{tabular}


System roll-back plan describes the events that may result in rolling back the transitioned system and putting the old system back to operation. Contingency plan lists all the possible scenarios that can lead to problems. For each possible unforeseen event, VAST Pakistan creates an action plan. Postdeployment monitoring plan includes activities to closely monitor the system working and performance while it is delivering its services. Cutover activity plan includes activities that need to be performed just before putting the system live. These include backup activities, sending system related alerts to relevant teams, diverting services to the backup system and the like. System outage plan describes the details of a timeframe when the system is not going to deliver its services any longer. The time-frame is planned and strictly followed to the precision in minutes. The deployment plans have to be executed within the allocated time-frame otherwise the outaged system can affect other operational areas.

Another important aspect of the deployment process at VAST Pakistan is the identification of all the system touchpoints and the teams impacted by problems in them. These teams include: (1) CRM team, (2) billing team, (3) middleware team, (4) VAS team, and (5) any other team where particular system touch-points are involved and have relevance to system architecture. These teams are involved in integration tests with their related interfaces and validate its connectivity and performance at the time of the deployment (see Activity DP5 in Table IV). They are also involved in monitoring the system on the provisional basis after deployment (see Activity DP6 in Table IV).

Finally, operations team is involved in installing the operational data (see Activity DP7 in Table IV). This activity is not the same as the data installation activity (see ME9 in Table II and Section IV.B). ME9 involves installing data on maintainer's site, while DP7 involves installing operational data on the customer's site. The operational data on the customer's site is the real-world data required for managing VAST Pakistan business. The data on the maintainer's site, on the other hand, is for the purpose of replicating the real-world data for facilitating the evolution and maintenance effort.

\section{E. Training}

VAST Pakistan performs most of the activities as suggested in the Training component as shown in Table V. However, it does not perform extra training activities like performing white-box testing, attending to modification requests and performing on-site support.

Training on the system, its structure and operation (see Activity T1 in Table V) is not considered as a crucial activity at VAST Pakistan. Since VAST Pakistan is the user and also $1^{\text {st }}$ and $2^{\text {nd }}$ line support provider, it does not need detailed system knowledge. The VAST Pakistan maintenance personnel mainly develop the system understanding during UAT, the system deployment, post-deployment provisional monitoring period and continuous communication with the vendor.

However, there may be some cases when the VAST Pakistan personnel need to be trained. For instance, when the VAST Pakistan personnel are unable to perform their duties based on current system knowledge and system's training
TABLE $V . \quad$ TRAINING. $\checkmark$ STANDS FOR PERFORMED. $\times$ STANDS FOR NOT PERFOMED. NEW STANDS FOR MISSING ACTIVITY IN $E M^{3}$ THE TAXONOMY

\begin{tabular}{|c|c|c|}
\hline $\mathrm{T} 1$ & Train on system, its structure and operation & $\checkmark$ \\
\hline $\mathrm{T} 1.1$ & $\begin{array}{l}\text { Create/update training material on system, its structure } \\
\text { and operation }\end{array}$ & $\checkmark$ \\
\hline $\mathrm{T} 1.2$ & Provide training on system, its structure and operation. & $\checkmark$ \\
\hline $\mathrm{T} 1.3$ & Attend to modification requests & $x$ \\
\hline $\mathrm{T} 1.4$ & Conduct white-box testing and debugging & $x$ \\
\hline $\mathrm{T} 2$ & Train on maintenance processes & $x$ \\
\hline $\mathrm{T} 2.1$ & $\begin{array}{l}\text { Create/update training material on maintenance } \\
\text { processes }\end{array}$ & $x$ \\
\hline $\mathrm{T} 2.2$ & Provide training on maintenance processes & $\mathbf{x}$ \\
\hline T3 & Train on system support processes & $\checkmark$ \\
\hline T3.1 & $\begin{array}{l}\text { Create/update training material on system support } \\
\text { process }\end{array}$ & $x$ \\
\hline T3.2 & Provide training on system support process & $\checkmark$ \\
\hline $\mathrm{T} 4$ & $\begin{array}{l}\text { Train on new technology to be used within operation } \\
\text { and maintenance }\end{array}$ & $\checkmark$ \\
\hline T4.1 & Create/update training material on new technology & $x$ \\
\hline T4.2 & Provide training on new technology & $\checkmark$ \\
\hline T5 & Provide on-site support in critical cases & $x$ \\
\hline \multicolumn{3}{|c|}{ New TRAINING ACTIVITIES } \\
\hline $\mathrm{T} 1.5$ & Participate in the system deployment & New \\
\hline T1.6 & Conduct UAT and post-deployment Testing for system & New \\
\hline T6 & Provide training on the deployment process & New \\
\hline T6.1 & $\begin{array}{l}\text { Prepare/update training material for the deployment } \\
\text { process }\end{array}$ & New \\
\hline T6.2 & $\begin{array}{l}\text { Develop a protocol for planning and executing the } \\
\text { deployment }\end{array}$ & New \\
\hline \multicolumn{3}{|c|}{ UPDATED TRAINING ACTIVITIES } \\
\hline T3.2 & \multicolumn{2}{|l|}{$\begin{array}{l}\text { Old: Provide training material on system support process } \\
\text { New: Provide training on system support process }\end{array}$} \\
\hline $\mathrm{T} 4.2$ & \multicolumn{2}{|l|}{$\begin{array}{l}\text { Old: Provide training material on new technology } \\
\text { New: Provide training on new technology }\end{array}$} \\
\hline
\end{tabular}

documentation, VAST Pakistan may request the vendor to provide system training. The vendor then updates the training material on the system, if required (see Activity T1.1 in Table $\mathrm{V}$ ) and provides training (see Activity T1.2 in Table V). The training material consists of training manuals on: (1) system's service logic, (2) system's architecture, and (3) system administration, installations and operations. VAST Pakistan bears all the training costs.

Since VAST Pakistan is the acquirer, it does not need any training on maintenance processes. However, it needs training on support processes (see Activities T2-T3 in Table V). It is VAST Pakistan's responsibility to provide training on the support processes to its support personnel. Usually, this form of training does not require major teaching effort. It is mainly conducted in form of interactive sessions and guidance to be provided to all the support personnel, including new hires and senior staff.

The training on new technology (see Activity T4 in Table V) is limited at VAST Pakistan. It only takes place in cases when technology for configuring and operating the systems gets changed. Being such a case, some of the operations team members explore the new technology and share it with the rest of the team.

Since VAST Pakistan only provides the $1^{\text {st }}$ and $2^{\text {nd }}$ line support, it does not need the detailed system knowledge. That is why, it neither attends to the modification requests nor does it perform the white box testing (see Activities T1.3-T1.4 in 
Table V) for the training purposes. Due to the fact that VAST Pakistan supports the system it uses, it by default provides onsite support, whether needed or not (see Activity T5 in Table V).

When evaluating the $E M^{3}$ taxonomy activities at VAST Pakistan, we observed that it lacked some of the activities. These are Participate in the system deployment, Conduct UAT and post-deployment testing for system, and Provide training on the deployment process.

Training is provided on the system deployment at VAST Pakistan (see Activity T6 in Table V). Preparatory sessions are executed on test systems to help planning and conducting the actual deployment. To facilitate the deployment, VAST Pakistan has a defined protocol to support the planning and execution of the deployment. This protocol or the guideline document focuses on a specific system and describes its prerequisites for the deployment, important considerations, tasks to be performed during deployment and the lessons learnt from the past system deployment experience. The difference between training material on deployment process and the guideline document is that the training is provided on general level for deployment process. The protocol, on the other hand, is a guideline document for a specific system such as a billing system.

The maintenance team can gain system knowledge by participating in the system deployment along with the system vendor. Also, system testing is being used as training activity at VAST Pakistan but not mentioned in the $E M^{3}$ taxonomy. We added new activities that suggest the maintainer to participate in the deployment process and perform the UAT and postdeployment system testing to gain the system knowledge (see Activities T1.5-T1.6 in Table V).

The operations team puts a lot of consideration and resources into planning and executing the deployment process. The planning itself takes around $75 \%$ of the overall deployment effort. Hence, a good protocol is pivotal for reducing the deployment cost. For this reason, we suggest that $E M^{3}$ covers the development of a protocol (see Activity T6.2 in Table V).

Finally, in the original $E M^{3}$ taxonomy, we detected a typo in the activities T3.2 and T4.2. We have re-phrased these activities as shown in Table $\mathrm{V}$.

\section{F. Documentation}

VAST Pakistan, being the acquirer and front-end supporter of the system, does not perform any of the activities suggested in the Documentation component, see Table VI. All documentation related activities are performed by the vendor.

\section{G. Maintainability}

VAST Pakistan, in its role of system acquirer and front-end

TABLE VI. DOCUMENTATION. A STANDS FOR ASSISTS THE VENDOR

\begin{tabular}{|l|l|l|}
\hline D1 & Establish a System Documentation Repository(SDR) & A \\
\hline D2 & Define services to be provided by the SDR & A \\
\hline D3 & Update SDR as per SCM & A \\
\hline D4 & Establish standards for documentation development & A \\
\hline
\end{tabular}

supporter, does not need to perform the activities for managing system maintainability (see Activity M1 in Table VII). System maintainability management is the responsibility of the vendor.

Regarding the data maintainability (see Activity M2 in Table VII), VAST Pakistan considers its future data processing and storage requirements and addresses the needs by placing requirements on system architecture. Distributed systems design is usually used in VAST Pakistan systems for data maintainability.

\section{FINAL REMARKS}

In this paper, we have evaluated the $E M^{3}$ taxonomy of handover activities in an industrial context. We did it from the perspective of the organization being both the acquirer of the system and provider of front-end support. Our goal was to evaluate the taxonomy's usefulness and credibility.

Our study has shown that most of the $E M^{3}$ handover activities are relevant in the studied context. The activities that were not relevant were the activities that were typically done by the back-end support. These include

- Transfer modification requests from development to maintenance (see Activity ME10 in Table II).

- $\quad$ Place modification requests in the MRR (see Activity ME11 in Table II).

- Attend to modification requests (see Activity T1.3 in Table V).

- Conduct white-box testing and debugging (see Activity T1.4 in Table V).

- $\quad$ Train on maintenance processes (see Activity T2 in Table V)

- $\quad$ Provide on-site support in critical cases (see Activity T5 in Table V).

- Assess system maintainability (see Activity M1 in table VII).

Our study has also revealed the need for more activities in the Deployment and Training components. Regarding the $E M^{3}$ Deployment component, it does not pay much attention to the deployment process itself. Our study has shown that the system deployment can be complex and hard to manage and that it strongly depends on the product type and organziation's business requirements. To address the deficiencies in the component, we added the following new activities:

- Plan for the system deployment (see Activity DP4 in Table IV) suggesting that the handover team prepare detailed plans for the deployment process. All the relevant teams should be identified and included in the planning. These plans include but are not limited to: (1) system rollback plan, (2) contingency plan, (3) post- deployment monitoring plan, (4) cutover activity plan, and (5) system outage plan.

TABLE VII. MAINTAINABILITY. $\checkmark$ STANDS FOR PERFORMED. $\times$ STANDS FOR NOT PERFOMED.

\begin{tabular}{|l|l|l|}
\hline M1 & Assess system maintainability & $\mathbf{x}$ \\
\hline M2 & Assess data maintainability & $\checkmark$ \\
\hline
\end{tabular}


- Install operational data (see Activity DP7 in Table IV) suggesting the installation of the operational data. The choice of the role performing this activity varies depending upon the system complexity and the organization's structure. In the simplest case, the maintenance team handles it. In the more complex cases, the database team handles this activity.

- Test the deployed system touch-points (see Activity DP5 in Table IV) suggesting that all the system touch-points be thoroughly tested. When a new system or system version is deployed, it is likely that it will operate and interact with its interfacing systems. For this reason, it is important that the owners of every touchpoint system check and validate connectivity and performance of their interfaces. This can save many problems later on. The choice of the roles involved in this activity varies on the nature of system. In the simplest case, maintenance team can test the system for related interfaces. In the more complex cases, the team concerning the touch-point performs the testing. The touchpoints may include but not limited to: CRM, billing, system monitoring and network services.

- Monitor the system operation: (see Activity DP6 in Table IV) suggesting the system should be closely monitored for a certain period of time when it is delivering the services after deployment. Maintenance team should execute the postdeployment monitoring plans and monitor the related interfaces for system trends and problems.

The training component does not mention the system deployment and testing as training activities. Also, transition team should be trained to perform the deployment. To address these deficiencies, we added the following new activities:

- Participate in the system deployment (see Activity T1.5 in Table V) suggesting that the parties involved in system support and maintenance should actively participate in the deployment process.

- Conduct UAT and post-deployment testing for system (see Activity T1.6 in Table V) suggesting that all the parties involved in deployment should perform UAT and postdeployment testing.

- Provide training on the deployment process (see Activity $\mathrm{T} 5$ in Table V) suggesting that the parties involved in the deployment process should be fully prepared to perform deployment.

- Develop a protocol for planning and executing the deployment (see Activity T5.2 in Table V) suggesting that the deployment team be provided with guidelines that describe the course of actions and activities to be performed when planning and executing the system deployment.

During our study, we also detected some anomalies. These concern activities T3.2: Provide training material on system support process and activity T4.2: Provide training material on new technology. These activities were redundant with the activities T3.1: Create/Update training material on system support process and T4.1: Create/Update training material on new technology respectively. We changed these to T3.2:
Provide training on system support process and T4.2: Provide training on new technology.

Finally, a number of activities in the $E M^{3}$ were redundant in the context of the organization studied. For this reason, we conclude that their choice mainly depends on the organization's or team's responsibility towards system. The choice of handover activities relevant for the organization depends on whether the organization is the acquirer, front-end supporter, back-end supporter, vendor or a combination of all these roles. Organization's size, its location and working culture and maturity may also determine the choice of the taxonomy activities.

\section{EPILOGUE}

Our evaluation of $E M^{3}$ taxonomy of handover activities has provided a new perspective on the handover process. In a similar vein as within our former studies [2][3][5], it has confirmed that the handover process strongly varies. Its design depends on product type, product lifecycle phase, the parties involved, databases, technology used, the types of system changes to be handed over and many other factors.

We believe that this study has provided a valuable feedback on system handover to the software community. However, more studies need to be made in order to find more information about the handover process and the factors affecting it.

\section{REFERENCES}

[1] A. S. Khan, M. Kajko-Mattsson, "Taxonomy of Handover Activities," in Proceedings of Software Maintenance Maturity Model Workshop (S3M'10), in press.

[2] A. S. Khan, M. Kajko-Mattsson, "Demarcating the Scope of a Handover Process," In Proceedings of International Conference on Software Engineering and Applications, IEEE, 2010, in press.

[3] M. Kajko-Mattsson, A. S. Khan , T. Tyrberg, "Evaluating A Taxonomy of Handover Activities in One Swedish Company," in Proceedings of 36th EUROMICRO Conference on Software Engineering and Advanced Applications (SEAA 2010), in press.

[4] M. Kajko-Mattsson, "Maturity Status within Front-End Support Organisations," In International Conference on Software Engineering, IEEE, Computer Society Press: Los Alamitos, CA, ISBN: 0-7695-28287, 2007.

[5] M. Kajko-Mattsson, LO. Gustafsson, "Cloud Outsourcing Requires A Proper Handover Process (Position Paper)," in Proceedings of International Conference on Advanced Information Management and Service, IEEE, 2010, in press.

[6] M. Kajko-Mattsson , "Towards a Business Maintenance Model, " in International Conference on Software Maintenance, IEEE Computer Society Press: Los Alamitos, CA, 2001, ISBN: 0-7695-1189-9, pp. 500509

[7] MIL-HDBK-347, Military Handbook, Mission critical computer resources software support, 22 May 1990. http://www.softwaresupportability.org/Docs/MIL-HDBK-347.pdf

[8] T. Pigoski, "Practical Software Maintenance," John Wiley \& Sons, 1997.

[9] T. Vollman, "Transitioning from development to maintenance," Proc. Software Maintenance conference, San Diego, CA, USA, 26-29 Nov 1990, ISBN: 0-8186-2091-9, pp. 189-199. 